\title{
Hydrodynamics with spin angular momentum from systematic coarse graining: a tutorial example
}

Article

Accepted Version

Ilg, P. (2016) Hydrodynamics with spin angular momentum from systematic coarse graining: a tutorial example. Journal of Non-Equilibrium Thermodynamics, 41 (2). pp. 89-97. ISSN 1437-4358 doi: https://doi.org/10.1515/jnet-2015-0067 Available at https://centaur.reading.ac.uk/57785/

It is advisable to refer to the publisher's version if you intend to cite from the work. See Guidance on citing.

To link to this article DOI: http://dx.doi.org/10.1515/jnet-2015-0067

Publisher: De Gruyter

All outputs in CentAUR are protected by Intellectual Property Rights law, including copyright law. Copyright and IPR is retained by the creators or other copyright holders. Terms and conditions for use of this material are defined in the End User Agreement.

www.reading.ac.uk/centaur

\section{CentAUR}


Central Archive at the University of Reading

Reading's research outputs online 


\title{
Research Article
}

Patrick IIg*

\section{Hydrodynamics with spin angular momentum from systematic coarse graining: A tutorial example}

\begin{abstract}
The derivation of time evolution equations for slow collective variables starting from a microscopic model system is demonstrated for the tutorial example of the classical, two-dimensional XY model. Projection operator techniques are used within a nonequilibrium thermodynamics framework together with molecular simulations in order to establish the building blocks of the hydrodynamics equations: Poisson brackets that determine the deterministic drift, the driving forces from the macroscopic free energy and the friction matrix. The approach is rather general and can be applied for deriving the equations of slow variables for a broad variety of systems.
\end{abstract}

Keywords: Coarse graining, projection operator, hydrodynamics, magnetization dynamics

DOI: 10.1515/jnet-YYYY-XXXX, Received ...; accepted ...

\section{Introduction}

Deriving coarse-grained equations from an underlying more microscopic model is not only important for practical purposes due to limited computational power, but also for better understanding those processes relevant for the phenomena under investigation $(1 ; 2)$. Hydrodynamics interpreted as the macroscopic dynamics of condensed matter systems is the coarsest level of description (3). Therefore, the derivation of closed hydrodynamic equations from underlying, most microscopic level of Hamilton mechanics has been studied for simple liquids for a long time $(4 ; 5)$. Peculiarities and related approaches for the solid state are discussed e.g. in (6). For complex fluids, however, this derivation remains a challenge due to additional structural variables that need to be included in the macroscopic description (7). Consequently, a wealth of phenomenological models have been put forward over the years. These models often lack a clear microscopic foundation which implies that (i) the parameters of the model need to be fitted and are often difficult to interpret physically and (ii) that the model cannot be improved in a systematic manner.

While many coarse-graining approaches have been proposed in the literature in the last years, relatively few of them are able to properly treat the nonequilibrium dynamics of the coarse-grained model (8). Irving and Kirkwood (4) presented a procedure for systematically deriving hydrodynamic balance equations from Hamiltonian dynamics. The Poisson bracket approach (9) builds on this work and derives the reversible part of the coarse-grained dynamics via a mapping of the microscopic Poisson bracket of classical mechanics. Their approach was applied by Stark and Lubensky to derive liquid-crystal hydrodynamics (10) and later generalized to biaxial molecules (11). While these approaches treat the reversible part of the coarse-grained dynamics very carefully, they are less systematic about irreversible contributions to the dynamics.

Here, I present an approach based on projection operator methods (12) embedded in a nonequilibrium thermodynamics framework to derive closed macroscopic equations starting from the underlying Hamiltonian dynamics in a systematic manner. In parts, this approach has already been applied to some example systems like rarefied gases, low-molecular polymer melts and liquid crystals $(13 ; 14 ; 15 ; 16 ; 17)$. This presentation follows the spirit of Chaikin and Lubensky (3) and illustrates the coarse-graining approach

*Corresponding Author: Patrick Ilg: Department of Mathematics \& Statistics, University of Reading, Reading RG6 6AX, UK 
for a simple, tutorial example of rotating dipoles where the hydrodynamics includes the intrinsic angular momentum and the magnetization dynamics. The present studies extends (3) in that dissipative effects are teated more carefully here. In a purely thermodynamic framework, hydrodynamics including spin angular momentum have been treated already in (18). A microscopic derivation of macroscopic models does not only deliver microscopic foundations of empirical models and microscopic expressions for the model parameters, but also allows to investigate modifications of model equations themselves $(8 ; 15)$.

\section{The XY Model}

Consider a system of $N$ identical, classical spins $\left\{\mathbf{u}_{i}\right\}, i=1, \ldots, N$ that are located on the nodes of a regular lattice. For simplicity, we consider a two-dimensional square lattice and restrict the spin orientations also to a two-dimensional plane. The microstate of the system is therefore defined by $z=\left(\theta_{1}, \ldots, \theta_{N}, l_{1}, \ldots, l_{N}\right) \in$ $\mathbb{R}^{2 N}$, where $l_{j}$ denotes the angular momenta of spin $j$ and the spin orientations are specified by $\mathbf{u}_{j}=$ $\left(\sin \theta_{j}, \cos \theta_{j}\right)$. The planar XY model is then defined by the Hamiltonian

$$
H(z)=\sum_{j=1}^{N} \frac{l_{j}^{2}}{2 I}-\frac{J}{2} \sum_{<i, j>} \cos \left(\theta_{i}-\theta_{j}\right)
$$

with $I$ the moment of inertia and the sum in the second term extends over all nearest neighbor pairs.

The equilibrium properties of the planar XY model have been studied extensively. At low temperatures, the model shows a BKT transition to a state with quasi long-range order (3). Here, I am not interested in (in fact exclude) the critical regime where XY model is usually studied in relation to phase transitions.

The microscopic time evolution in classical mechanics is given by Hamilton's equations of motion,

$$
\dot{\theta}_{i}=\frac{\partial H}{\partial l_{i}}=l_{i} / I, \quad i_{i}=-\frac{\partial H}{\partial \theta_{i}}=-J \sum_{j(\mathrm{nn} i)} \sin \left(\theta_{i}-\theta_{j}\right)
$$

The dynamics can equivalently be expressed by $\frac{\mathrm{d}}{\mathrm{d} t} A=\{A, H\}$ with the microscopic Poisson bracket of two functions $A(z), B(z)$ defined by

$$
\{A, B\}=\sum_{j=1}^{N}\left(\frac{\partial A}{\partial \theta_{j}} \frac{\partial B}{\partial l_{j}}-\frac{\partial A}{\partial l_{j}} \frac{\partial B}{\partial \theta_{j}}\right) .
$$

The Poisson bracket (3) satisfies the anti-symmetry condition, $\{A, B\}=-\{B, A\}$, the Leibniz rule, $\{A B, C\}=A\{B, C\}+\{A, C\} B$, and the Jacobi identity, $\{A,\{B, C\}\}+\{B,\{C, A\}\}+\{C,\{A, B\}\}=0(3)$.

On a macroscopic level, we want to describe the system in terms of a few collective variables only. The set of collective variables should include (i) densities of conserved quantities and (ii) broken symmetry variables. In the present example, the planar XY model has only two conserved quantities: the total energy $E=H$ and the total angular momentum $L=\sum_{j=1}^{N} l_{j}$. To be able to describe also the partial orientational ordering at low temperatures, we include the magnetization $\mathbf{m}=(\sin \theta, \cos \theta)$ in the set of collective variables. We therefore define the corresponding densities as $(4 ; 5)$

$$
\Pi_{\varepsilon}(\mathbf{r}, t)=\sum_{j=1}^{N} \varepsilon_{j}(t) \chi\left(\mathbf{r}-\mathbf{r}_{j}\right), \quad \Pi_{\ell}(\mathbf{r}, t)=\sum_{j=1}^{N} l_{j}(t) \chi\left(\mathbf{r}-\mathbf{r}_{j}\right), \quad \Pi_{\mathbf{m}}(\mathbf{r}, t)=\sum_{j=1}^{N} \mathbf{u}_{j}(t) \chi\left(\mathbf{r}-\mathbf{r}_{j}\right),
$$

where the energy per spin is defined by $\varepsilon_{j}=\frac{l_{j}^{2}}{2 I}-\frac{J}{2} \sum_{i(\mathrm{nn} j)} \cos \left(\theta_{i j}\right), \theta_{i j}=\theta_{i}-\theta_{j}$, and the sum includes all nearest neighbors of spin $j$. Introducing a non-hydrodynamic collective variable to describe orientational ordering is also done in other systems, like magnetic fluids (19) and liquid crystals (3; 20;21). Equations (4) define mappings from the instantaneous microscopic configurations to the set of collective variables. Note that we have introduced a weight function $\chi(\mathbf{r})$ that can be thought of as a smoothed Dirac delta function. The precise form of the function $\chi$ should be irrelevant in the following. The challenge we want to address is how to derive closed equations for the average collective variables. 


\section{Mori-Zwanzig approach}

Any statistical coarse-graining approach needs to introduce an appropriate ensemble in order to mediate the transition from the microscopic to the macroscopic level of description. Here, in particular, we need to calculate average values of the collective variables $x_{k}(t)=\int \mathrm{d} z \Pi_{k}(z) \rho(z ; t)$. In principle, the probability density can be obtained from the Liouville equation $\partial_{t} \rho=-\{\rho, H\}$. However, exact solutions for $\rho(z ; t)$ are not available unless the microscopic system is fully solved. Therefore, we resort to the so-called relevant ensemble $\rho^{*}$ in order to calculate approximate values for average quantities. Different choices for the relevant ensemble can be made. In most cases, the maximum entropy principle is invoked (22),

$$
S[\rho]=-k_{\mathrm{B}} \int \mathrm{d} z \rho(z) \ln \rho(z) \rightarrow \max , \quad x_{k}[\rho]=\int \mathrm{d} z \Pi_{k}(z) \rho(z) \text { fixed. }
$$

The solution to the constraint maximization problem is given by the generalized canonical ensemble

$$
\rho^{*}(z)=\frac{1}{\Xi} \exp \left[-\sum_{k} \Lambda_{k} \Pi_{k}(z)\right]
$$

where the Lagrange multipliers $\Lambda=\Lambda(x)$ are determined from the constraints $x_{k}=\int \mathrm{d} z \Pi_{k}(z) \rho^{*}(z)$. The partition function $\Xi(\Lambda)$ ensures the normalization of $\rho^{*}$,

$$
\Xi(\Lambda)=\int \mathrm{d} z \exp \left[-\sum_{k} \Lambda_{k} \Pi_{k}(z)\right] .
$$

The macroscopic entropy associated with the relevant ensemble $(5)$ is defined by $S^{*}=S\left[\rho^{*}\right]$. Upon inserting the particular form of $\rho^{*}$ in the Gibbs entropy one finds that $S^{*}$ is given by the Legendre transform

$$
S^{*}(x) / k_{\mathrm{B}}=\ln \Xi+\sum_{k} \Lambda_{k} x_{k}
$$

Noting that $\frac{\partial \ln \Xi}{\partial \Lambda_{k}}=-x_{k}$, the Lagrange multipliers are thermodynamically conjugate variables to $x$,

$$
k_{\mathrm{B}} \Lambda_{k}=\frac{\partial S^{*}}{\partial x_{k}} .
$$

Introducing the relevant ensemble for averaging is, however, not sufficient for coarse-graining when starting from a microscopic system, as the averaged dynamics is still non-dissipative, i.e. the Gibbs entropy $S[\rho]$ is preserved in Hamiltonian dynamics. Thus, one needs to treat the dynamics orthogonal to manifold spanned by the relevant ensemble. We will employ here the projection operator approach proposed by Mori and Zwanzig. The approach is sketched here only briefly, more details can be found in Refs. $(12 ; 22 ; 23 ; 24)$. Consider the time evolution of a phase space function $A(z)$ written as $\dot{A}=\mathcal{L} A$, with the Liouville operator $\mathcal{L} A=\{A, H\}$. Formally, the phase space function evaluated at time $t$ is given by $A(z ; t)=e^{t \mathcal{L}} A(z)$. In order to decompose the microscopic dynamics, define the projector $\mathbb{P}$ on the subspace of phase space functions of interest, that are spanned by the set of collective variables. The operator identity (12)

$$
e^{\mathcal{L} t}=e^{\mathcal{L} t} \mathbb{P}(t)+\int_{0}^{t} \mathrm{~d} s e^{\mathcal{L} s} \mathbb{P}(s)[\mathcal{L}-\dot{\mathbb{P}}(s)] \mathbb{Q}(s) G(s, t)+\mathbb{Q}(0) G(0, t)
$$

with time-ordered exponential $G(u, t)=\mathcal{T} \exp \left[\int_{u}^{t} \mathrm{~d} s \mathbb{Q}(s) \mathcal{L} \mathbb{Q}(s)\right]$ plays a central role in the projection operator approach. Note that the projectors $\mathbb{P}$ and $\mathbb{Q}=1-\mathbb{P}$ are time-dependent since the collective variables evolve in time. Applying Eq. (9) to $\mathcal{L} \Pi_{k}$ and averaging with the relevant ensemble, we arrive at the exact transport equation for the collective variables

$$
\frac{\mathrm{d}}{\mathrm{d} t} x_{k}(t)=\left\langle\mathcal{L} \Pi_{k}\right\rangle_{x(t)}+\sum_{j} \int_{0}^{t} \mathrm{~d} s\left\langle\left[\mathcal{L} \Pi_{j}\right] \mathbb{Q}(s) G(s, t) \mathcal{L} \Pi_{k}\right\rangle_{x(s)} \Lambda_{j}(x(s)) .
$$


In Eq. (10) use has been made of the generalized canonical ensemble (5) and Grabert's form of the projector. The first term in Eq. (10) is the deterministic drift, $v_{k}=\left\langle\mathcal{L} \Pi_{k}\right\rangle_{x}=\left\langle\left\{\Pi_{k}, H\right\}\right\rangle_{x}=\int \mathrm{d} z \rho_{x}^{*}(z) \mathcal{L} \Pi_{k}$, obtained by averaging the microscopic time derivative with the relevant ensemble corresponding to the actual values of the collective variables. The second term in Eq. (10) involves not only the projector orthogonal to the subspace spanned by the collective variables but also memory integrals. For Grabert's projector, the term proportional to $\dot{\mathbb{P}}$ does not contribute (22). Note also that the last term in Eq. (9) does not contribute to averages with the relevant ensemble.

In order to make further progress, we make a crucial step and adopt the Markovian approximation. In particular, we assume a separation of time scales such that the collective variables evolve only on times larger than a separating time scale $\tau_{\mathrm{s}}$, whereas correlations between the orthogonal components decay on time scales short then $\tau_{\mathrm{s}}$. With this assumption, the memory integral collapses and Eq. (10) simplifies to

$$
\frac{\mathrm{d}}{\mathrm{d} t} x_{k}(t)=\left\langle\left\{\Pi_{k}, H\right\}\right\rangle_{x(t)}+\sum_{j} M_{k j}(x(t)) \Lambda_{j}(x(t)), \quad M_{j k}(x)=\int_{0}^{\tau_{\mathrm{s}}} \mathrm{d} t\left\langle\dot{\Pi}_{k}^{\mathrm{f}}(t) \dot{\Pi}_{j}^{\mathrm{f}}(0)\right\rangle_{x}
$$

with $\dot{\Pi}_{k}^{\mathrm{f}}(t)=e^{\mathbb{Q} \mathcal{L} t} \mathbb{Q} \mathcal{L} \Pi_{k}$ the fast part of the time evolution. When we additionally impose the natural restriction that the total energy $E$ shall be available also on the macroscopic level of description, $H(z)=$ $E(\Pi(z))$, the deterministic drift can be written as $v_{k}=\sum_{j}\left\langle\left\{\Pi_{k}, \Pi_{j}\right\}\right\rangle \frac{\partial E}{\partial x_{j}}$ and Eq. (11) is of the GENERIC form (22). The anti-symmetry property and Leibniz rule for the Poisson matrix $L_{k j}=\left\langle\left\{\Pi_{k}, \Pi_{j}\right\}\right\rangle$ is evident; the Onsager-Casimir symmetry of the friction matrix $M_{j k}$ is also readily shown from microscopic reversibility. Only the validity of the Jacobi identity of the Poisson matrix is not obvious (23).

\section{Deriving the Macroscopic Entropy}

Coming back to the XY model, our first aim is to derive an explicit expression for the macroscopic $S^{*}(x)$ in order to calculate the driving forces $\Lambda_{k}$, Eq. (8), entering the dynamics of the collective variables (11). We prefer to determine $S^{*}$ first since the Lagrange multipliers $\Lambda_{k}$ have to respect Maxwell relations, $\partial \Lambda_{k} / \partial x_{j}=\partial \Lambda_{j} / \partial x_{k}$, which are automatically satisfied once $S^{*}(x)$ is established. For our choice of collective variables for the XY model, the partition function of the generalized canonical ensemble (5) reads

$$
\Xi[\beta, \lambda, \mathbf{h}]=\int \mathrm{d} z \exp \left[-\sum_{j=1}^{N}\left(\beta_{j} \varepsilon_{j}+\lambda_{j} l_{j}+\mathbf{h}_{j} \cdot \mathbf{u}_{j}\right)\right],
$$

with $\beta_{j}=\int \mathrm{d}^{d} r \beta(\mathbf{r}) \chi\left(\mathbf{r}-\mathbf{r}_{j}\right)$ the (smoothed) value of the Lagrange multiplier at position of spin $j$ and

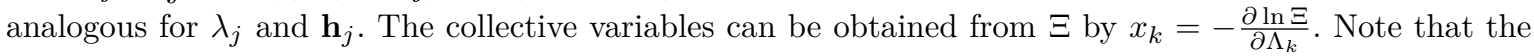
integration over angular momenta can be performed exactly with the help of Gaussian integrals, leading to $\Xi[\beta, \lambda, \mathbf{h}]=\Xi^{\mathrm{kin}}[\beta, \lambda] \Xi^{\operatorname{conf}}[\beta, \mathbf{h}]$ with $\Xi^{\mathrm{kin}}[\beta, \lambda]=\exp \left[\frac{1}{2} \sum_{j=1}^{N}\left(\ln \left[2 \pi I \beta_{j}\right]+I \lambda_{j}^{2} / \beta_{j}\right)\right]$. We therefore find $\ell(\mathbf{r})=-I \lambda(\mathbf{r}) / T(\mathbf{r})$. The splitting of $\Xi$ implies a corresponding splitting of the entropy, $S^{*}=S^{\mathrm{kin}}+S^{\mathrm{conf}}$. The configurational part, however, cannot be calculated exactly. Here, I will first briefly sketch the meanfield calculation and then comment on a more accurate but numerical computation. Define the matrix $J_{i j}$ to be equal to $J$ if $i$ and $j$ are nearest neighbors and zero else. With the help of a Hubbard-Stratonovich transformation, one obtains $\Xi^{\text {conf }}=\int \mathrm{D} \phi e^{-\mathcal{S}[\phi]}$ where $\mathrm{D} \phi=\prod_{i=1}^{N} \frac{\mathrm{d} \phi_{i}}{\sqrt{2 \pi}}$ and the action $\mathcal{S}$ is defined by

$$
\mathcal{S}[\phi]=-\frac{1}{2} \ln \operatorname{det}(\beta J)+\frac{1}{2} \sum_{i j} \beta_{i} J_{i j} \phi_{i} \cdot \phi_{j}+\sum_{i=1}^{N} \mathcal{S}_{0}\left(\mathbf{h}_{i}-\beta_{i} \sum_{j} J_{i j} \phi_{j}\right),
$$

where the $i j$-th component of the matrix $\beta J$ is $\beta_{i} J_{i j}$ (no summation convention). The action for the noninteraction system is $\mathcal{S}_{0}(\mathbf{h})=\ln \left[2 \pi I_{0}(h)\right]$ and $I_{n}(x)$ the modified Bessel function of the first kind of order $n$. In the mean-field approximation, the partition function is approximated by $\Xi^{\operatorname{conf}} \approx e^{-\mathcal{S}[\bar{\phi}]}$ with the 
saddle point $\bar{\phi}_{i}=\mathbf{m}_{i}$. For the homogeneous case, $\mathbf{h}_{i}=\mathbf{h}, \mathbf{m}_{i}=\mathbf{m}$, one finds from $\Xi^{\text {conf }}$ and Eq. (7)

$$
S^{\operatorname{conf}}(\mathbf{m}) / N k_{\mathrm{B}}=\frac{1}{2} \ln \operatorname{det}(\beta J)-\frac{1}{2} K \mathbf{m}^{2}+\ln \left[I_{0}(\mathbf{h}-K \mathbf{m})\right]-\mathbf{h} \cdot \mathbf{m},
$$

with $\mathbf{h}=\mathbf{h}(\mathbf{m})$ and $K=c \beta J$ the dimensionless coupling parameter and $c$ the coordination number of the lattice ( $c=4$ for a two-dimensional square lattice). The magnetization $\mathbf{m}$ is parallel to the Lagrange multiplier $\mathbf{h}$ and their magnitudes are related by $m=-K m+\frac{I_{1}(h-K m)}{I_{0}(h-K m)}$. A more explicit expression can be obtained in the limit of weak ordering,

$$
S^{\operatorname{conf}}(\mathbf{m}) / N k_{\mathrm{B}} \approx-\left(1+\frac{3}{2} K-K^{2}\right) \mathbf{m}^{2}-\frac{1}{4}(1-3 K)(1+K)^{3} \mathbf{m}^{4}-\frac{5}{36}(1-25 K)(1+K)^{5} \mathbf{m}^{6}+\ldots
$$

It is worth emphasizing that Eq. (14) is not a low-order polynomial but diverges logarithmically near the fully oriented state. This is in sharp contrast to the usual Ginzburg-Landau free energy which is a quartic function that becomes unphysical for strong ordering (25). It should also be mentioned that the continuous transition suggested by Eq. (15) is an artifact of the mean-field approximation, as the planar XY model shows a Beresinskii-Kosterlitz-Thouless transition instead (3).

Finally, I want to mention that a more accurate way of determining $S^{\text {conf }}$ can be obtained as follows. Using the property that $\Lambda_{k}$ are thermodynamically conjugate variable to $x_{k}$, we can integrate Eq. (8) in order to find the entropy change from the reference state $S_{0}^{*}$ corresponding to $\Lambda=0$,

$$
S^{*}(x)-S_{0}^{*}=k_{\mathrm{B}} \sum_{k} \int_{0}^{x} \Lambda_{k} \mathrm{~d} x_{k} \Rightarrow S^{\operatorname{conf}}(\mathbf{m})=\int_{0}^{\mathbf{m}} \mathbf{h} \cdot \mathrm{d} \mathbf{m} .
$$

We can therefore determine $\mathbf{m}(\mathbf{h})=\langle\mathbf{u}\rangle$ from Monte-Carlo simulations in the generalized canonical ensemble for various values of $\mathbf{h}$ and use these in Eq. (16) in order to arrive at numerical values for the orientational contribution to the entropy $S^{\operatorname{conf}}(\mathbf{m})$. We have carried out this procedure for different models of liquid crystals in Refs. $(26 ; 25 ; 16)$, where we have also shown how to reconstruct the functional form of $S^{\operatorname{conf}}(\mathbf{m})$ from the numerical data. This last step is not necessary, as one could also work e.g. with tabulated values for the driving forces $\Lambda_{k}$. An explicit functional form, however, is more enlightening and also more convenient for later use in the coarse-grained model.

\section{Poisson brackets}

From the projection operator derivation, Eq. (11), one finds that the deterministic drift $v_{k}$ is given by $v_{k}=\left\langle\left\{\Pi_{k}, H\right\}\right\rangle$, where averages are performed with the relevant ensemble $\rho^{*}$. To derive the corresponding Poisson brackets, we first find the instantaneous conservation laws from the definitions of the mappings (4)

$$
\dot{\Pi}_{\varepsilon}(\mathbf{r}, t)=-\frac{\partial}{\partial \mathbf{r}} \cdot \hat{\boldsymbol{\jmath}}(\mathbf{r}, t), \quad \dot{\Pi}_{\ell}(\mathbf{r}, t)=-\frac{\partial}{\partial \mathbf{r}} \cdot \hat{\boldsymbol{\tau}}(\mathbf{r}, t)
$$

with explicit microscopic expressions for the energy and angular momentum flux,

$$
\hat{\boldsymbol{j}}(\mathbf{r}, t)=\frac{1}{2 I} \sum_{<i, j>} \tilde{l}_{i j} \mathbf{r}_{i j} F_{i j} \chi_{i j}(\mathbf{r}), \quad \hat{\boldsymbol{\tau}}(\mathbf{r}, t)=\frac{1}{2} \sum_{<i, j>} \mathbf{r}_{i j} F_{i j} \chi_{i j}(\mathbf{r}) .
$$

In Eq. (18) we have introduced the torque exerted by $\operatorname{spin} j$ on $i, F_{i j}=-J \sin \left(\theta_{i j}\right), \tilde{l}_{i j}=l_{i}+l_{j}$ and the short notation for smearing out the weight between spins $i$ and $j, \chi_{i j}(\mathbf{r})=\int_{0}^{1} \mathrm{~d} s \chi\left(\mathbf{r}-\mathbf{r}_{i}+s \mathbf{r}_{i j}\right)$. The derivation of Eqs. $(17,18)$ is analogous to the procedure suggested by Irving and Kirkwood (4). In the present case, there is no deterministic flux of energy, $v_{\varepsilon}=0$. In the isotropic state also $v_{\ell}$ vanishes. But for spatially varying orientational ordering, we may expand $\sin \left(\theta_{i j}\right) \approx \theta_{i j}$ in $\hat{\boldsymbol{\tau}}$ and arrive at $v_{\ell}=k_{\text {eff }}\left|\mathbf{m} \times \nabla^{2} \mathbf{m}\right|=k_{\text {eff }} \nabla^{2} \theta$, where $k_{\mathrm{eff}}=c J a^{2}$. It is worth to note that we arrive at the same expression for $v_{\ell}$ by the GENERIC route of calculating $v_{l}=\left\langle\left\{\Pi_{\ell}, \Pi_{\mathbf{m}}\right\}\right\rangle \cdot \frac{\delta E}{\delta \mathbf{m}}$ with the Poisson bracket $\left\langle\left\{\Pi_{\ell}, \Pi_{\mathbf{m}}\right\}\right\rangle=\mathbf{m} \times \mathbf{e}_{z} \delta\left(\mathbf{r}-\mathbf{r}^{\prime}\right)$. For spatially 
varying orientational order, we employ the frequently used square gradient approximation (3) so that the macroscopic energy becomes $E(x)=\int \mathrm{d}^{d} r\left[\varepsilon+\frac{1}{2} k_{\mathrm{eff}}(\nabla \mathbf{m})^{2}\right]$. Note that the second, elastic contribution to $E$ is the dominant term in the spin wave approximation (3).

Consider now the deterministic drift of the magnetization, $v_{\mathbf{m}}=\left\langle\left\{\Pi_{\mathbf{m}}, H\right\}\right\rangle$. Since the magnetization is not conserved, we do not expect a local conservation law for $\Pi_{\mathbf{m}}$. Indeed, straightforward calculation gives $\left\{\Pi_{\mathbf{m}}, H\right\}=\sum_{j} \mathbf{u}_{j} \times \mathbf{e}_{z} \frac{l_{j}}{I} \chi\left(\mathbf{r}-\mathbf{r}_{j}\right)$. Performing the averages leads to

$$
v_{\mathbf{m}}=\boldsymbol{\Omega} \times \mathbf{m}
$$

with the angular velocity $\boldsymbol{\Omega}(\mathbf{r})=(0,0, \omega(\mathbf{r}))$ with $\omega(\mathbf{r})=I^{-1} \ell(\mathbf{r})$. Note that Eq. (19) defines the corotational derivative of the magnetization with respect to the mean angular velocities of the spins.

\section{Friction matrix from correlated fluctuations}

The projection operator derivation provides with Eq. (11) a formal expression for evaluating the elements of the friction matrix $M_{k j}$. As is often done, we approximate the time evolution involving the orthogonal projector by the fast, fluctuating part of the true dynamics, $\dot{\Pi}_{k}^{\mathrm{f}} \approx e^{\mathcal{L} t} \mathbb{Q} \mathcal{L} \Pi_{k}$. This approximation can be justified for short enough times (24). Then, the resulting Green-Kubo type expressions can be evaluated using Molecular Dynamics simulations. It is important to emphasize that these simulations need to be performed only up to the separating time scale $\tau_{\mathrm{s}}$, i.e. very short on the time scale of the collective variables. For the diagonal components in our tutorial example, Eq. (11) becomes

$$
k_{\mathrm{B}} M_{\varepsilon \varepsilon}=\frac{\partial}{\partial r_{\alpha}} \frac{\partial}{\partial r_{\beta}^{\prime}} \int_{0}^{\tau_{\mathrm{s}}} \mathrm{d} t\left\langle\hat{\jmath}_{\alpha}(\mathbf{r}, t) \hat{\jmath}_{\beta}\left(\mathbf{r}^{\prime}, 0\right)\right\rangle_{x}, \quad k_{\mathrm{B}} M_{\ell \ell}=\frac{\partial}{\partial r_{\alpha}} \frac{\partial}{\partial r_{\beta}^{\prime}} \int_{0}^{\tau_{\mathrm{s}}} \mathrm{d} t\left\langle\tau_{\alpha}(\mathbf{r}, t) \tau_{\beta}\left(\mathbf{r}^{\prime}, 0\right)\right\rangle_{x}
$$

and $k_{\mathrm{B}} M_{\mathbf{m m}}=\int_{0}^{\tau_{\mathrm{s}}} \mathrm{d} t \sum_{i j}\left\langle\dot{\mathbf{u}}_{i}^{\mathrm{f}}(t) \dot{\mathbf{u}}_{j}^{\mathrm{f}}(0) \chi\left(\mathbf{r}-\mathbf{r}_{i}\right) \chi\left(\mathbf{r}^{\prime}-\mathbf{r}_{j}\right)\right\rangle_{x}$. The fluxes are defined in Eq. (18), where now $\tilde{l}_{i j}=l_{i}+l_{j}-\ell\left(\mathbf{r}_{i}\right)-\ell\left(\mathbf{r}_{j}\right)$ denotes the deviation from the mean angular momentum and $\dot{\mathbf{u}}_{i}^{\mathrm{f}}=$ $\left(\cos \theta_{i},-\sin \theta_{i}\right)^{T} \frac{l_{j}}{I}-v_{\mathbf{m}}\left(\mathbf{r}_{i}\right)$. From the off-diagonal components, only $M_{\varepsilon \mathbf{m}}=-\frac{\partial}{\partial \mathbf{r}} \cdot \mathbf{R}$ and $M_{\mathbf{m} \varepsilon}=\mathbf{R}^{T} \cdot \frac{\partial}{\partial \mathbf{r}}$ are non-zero with $\mathbf{R}=\int_{0}^{\tau_{\mathrm{s}}} \mathrm{d} t \sum_{j}\left\langle\hat{\boldsymbol{\jmath}}(\mathbf{r}, t) \dot{\mathbf{u}}_{j}^{\mathrm{f}}(0) \chi\left(\mathbf{r}^{\prime}-\mathbf{r}_{j}\right)\right\rangle_{x}$. Other cross-couplings vanish, as can be shown explicitly by averaging over the peculiar angular momenta. The same conclusion can be drawn by observing that the friction matrix couples only quantities with the same behavior under time reversal (27). Since $\varepsilon$ and $\mathbf{m}$ are even and $\ell$ is odd under time reversal, the only allowed cross-coupling is between $\varepsilon$ and $\mathbf{m}$.
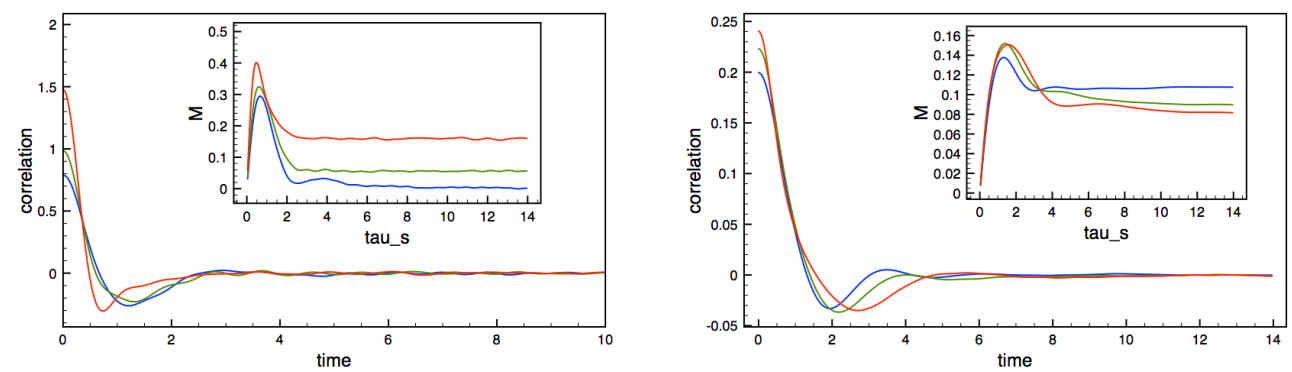

Fig. 1. The main panels show the correlation functions $\left\langle\dot{\mathbf{u}}_{i}^{\mathrm{f}}(t) \cdot \dot{\mathbf{u}}_{i}^{\mathrm{f}}(0)\right\rangle$ (left panel) and $\langle\hat{\tau}(t) \hat{\tau}(0)\rangle$ (right panel) obtained from Molecular Dynamics simulations of the XY model on a $100 \times 100$ square grid for different values of $\varepsilon$ in the high temperature phase. The insets show the corresponding time integrals which are proportional to $M_{m m}$ and $M_{\ell \ell}$, respectively.

Except near phase transitions, the spatial correlations are expected to be short-ranged so that on a macroscopic scale, we can approximate the matrix components with expressions that are local in space, $M_{\varepsilon \varepsilon}\left(\mathbf{r}, \mathbf{r}^{\prime}\right) \approx \frac{\partial}{\partial \mathbf{r}} \cdot \boldsymbol{\kappa}(\mathbf{r}) \cdot \frac{\partial}{\partial \mathbf{r}^{\prime}} \delta\left(\mathbf{r}-\mathbf{r}^{\prime}\right), M_{\ell \ell}\left(\mathbf{r}, \mathbf{r}^{\prime}\right) \approx \frac{\partial}{\partial \mathbf{r}} \cdot \boldsymbol{\Gamma}(\mathbf{r}) \cdot \frac{\partial}{\partial \mathbf{r}^{\prime}} \delta\left(\mathbf{r}-\mathbf{r}^{\prime}\right)$, and $M_{\mathbf{m m}}\left(\mathbf{r}, \mathbf{r}^{\prime}\right) \approx \mathbf{D}(\mathbf{r}) \delta\left(\mathbf{r}-\mathbf{r}^{\prime}\right)$. 
The transport coefficients $\boldsymbol{\kappa}, \boldsymbol{\Gamma}$ and $\mathbf{D}$ are related to the thermal conductivity, angular momentum and spin diffusivity, respectively. Decomposing the friction matrix $M=C \cdot D_{M} \cdot C^{T}$ with $C=\operatorname{diag}(\nabla, \nabla,-\mathbf{1})$, we find that the matrices $\boldsymbol{\kappa}, \boldsymbol{\Gamma}, \mathbf{D}$ and $\left(\begin{array}{cc}\boldsymbol{\kappa} & \mathbf{R} \\ \mathbf{R}^{T} & \mathbf{D}\end{array}\right)$ need to be positive semi-definite in order to ensure a proper friction matrix that in turn ensures non-negativity of the entropy production (22).

In Fig. 1, correlation functions $\left\langle\dot{\mathbf{u}}_{i}^{\mathrm{f}}(t) \cdot \dot{\mathbf{u}}_{i}^{\mathrm{f}}(0)\right\rangle$ (left panel) and $\langle\hat{\tau}(t) \hat{\tau}(0)\rangle$ are shown as a function of time $t$ for various values of $\varepsilon$ in the high-temperature phase and in the absence of an external field, $\mathbf{h}=0$. The inset shows $\Gamma$ as a function of $\tau_{\mathrm{s}}$. The plateau indicates the existence of a separating time scale between fast dynamics of fluctuating quantities and slow time evolution of the chosen collective variables.

\section{Hydrodynamic equations}

Inserting the above results for the deterministic drift and the local form of the friction matrix into the general transport equation (11), the time evolution equations for the hydrodynamic fields become

$$
\begin{aligned}
\frac{\partial}{\partial t} \varepsilon & =-\frac{\partial}{\partial \mathbf{r}} \cdot \boldsymbol{\kappa} \cdot \frac{\partial}{\partial \mathbf{r}} \frac{1}{T}-\frac{\partial}{\partial \mathbf{r}} \cdot \mathbf{R} \cdot \mathbf{h} \\
\frac{\partial}{\partial t} \ell & =k_{\mathrm{eff}} \nabla^{2} \theta+\frac{\partial}{\partial \mathbf{r}} \cdot \boldsymbol{\Gamma} \cdot \frac{\partial}{\partial \mathbf{r}} \frac{\omega}{T} \\
\frac{\partial}{\partial t} \mathbf{m} & =\boldsymbol{\Omega} \times \mathbf{m}+\mathbf{R}^{T} \cdot \frac{\partial}{\partial \mathbf{r}} \frac{1}{T}+\mathbf{D} \cdot \mathbf{h}
\end{aligned}
$$

The transport coefficients $\boldsymbol{\kappa}, \mathbf{R}, \boldsymbol{\Gamma}, \mathbf{D}$ are of the general form $\mathbf{A}=A^{\|} \mathbf{n n}+A^{\perp}(\mathbf{1}-\mathbf{n n})$ where $\mathbf{1}$ denotes the two-dimensional unit matrix and $\mathbf{n}=\mathbf{m} / m$. In the high temperature, isotropic phase $\mathbf{A}=A \mathbf{1}$ and positivity of the friction matrix is ensured by $\kappa>0, \Gamma>0, D>0$ and $\kappa D>R^{2}$. For low temperatures, the $\mathrm{XY}$ model shows only quasi-long range order in the absence of an external field. A mean-field approximation is therefore not adequate in this regime.

To summarize, the main assumptions needed to arrive at Eqs.(21-23) are (i) existence of a well-defined entropy function $S^{*}(x)$ on the macroscopic level defined by $x=(\varepsilon, \ell, \mathbf{m})$, (ii) the generalized canonical ensemble to provide good approximations for averages needed on the macroscopic level, (iii) Markovian approximation valid for times $t>\tau_{\mathrm{s}}$, (iv) weak spatial variations on macroscopic scales $r \gg a$, and (v) approximate calculations of the friction matrix by short time fluctuations rather than the orthogonal dynamics $\mathbb{Q} \mathcal{L}$. The form of the resulting equations is as expected (3): the energy balance equation (21) with heat conduction $\boldsymbol{\kappa}$ and a cross-term due to orientation coupling; the angular momentum balance equation (22) with spin viscosity $\boldsymbol{\Gamma}$ and an elastic contribution $k_{\text {eff }}$; and the magnetization equation (23) with the convective derivative, the relaxation term and a cross coupling in the presence of temperature gradients. The latter known as spin Seebeck effect has received considerable interest in recent years (28).

\section{Conclusions}

The projection operator formalism embedded in a nonequilibrium thermodynamics framework provides a powerful tool for systematic coarse-graining the microscopic dynamics to the macroscopic level. In the approach suggested here, analytical calculations are complemented by guided molecular simulations that are used to reconstruct missing building blocks of the macroscopic model. In particular, Monte-Carlo simulations in the generalized canonical ensemble are performed in order to obtain the macroscopic entropy, whereas short Molecular Dynamics simulations are employed to determine transport coefficients entering the friction matrix. The approach is illustrated for a simple, tutorial example but is rather general and should be useful for a wide variety of systems for which the set of slow collective variables is known.

Acknowledgment: The author has greatly benefitted from discussions with H.C. Öttinger and M. Kröger. 
Funding: PI acknowledges support from a career integration grant of the European Union, CIG-631233.

\section{References}

[1] P. Español. Statistical mechanics of coarse-graining. In Novel Methods in Soft Matter Simulations, volume 640 of Lecture Notes in Physics, pages 69-115. Springer, 2004.

[2] C. Peter and K. Kremer. Multiscale simulation of soft matter systems - from the atomistic to the coarse-grained level and back. Soft Matter, 5:4357-4366, 2009.

[3] P. M. Chaikin and T. C. Lubensky. Principles of Condensed Matter Physics. Cambridge University Press, Cambridge, 1995.

[4] J. H. Irving and J. G. Kirkwood. The statistical mechanical theory of transport processes. IV. The equations of hydrodynamics. J. Chem. Phys., 18:817-829, 1950.

[5] J. J. de Pablo and H. C. Öttinger. An atomistic approach to general equation for the nonequilibrium reversibleirreversible coupling. J. Non-Newtonian Fluid Mech., 96:137-162, 2001.

[6] N. C. Admal and B. Tadmor. A unified description of stress in molecular systems. J. Elast., 100:63-143, 2000.

[7] R. G. Larson. The Structure and Rheology of Complex Fluids. Oxford University Press, Oxford, UK, 1999.

[8] P. Ilg, V. G. Mavrantzas, and H. C. Öttinger. Multiscale modeling and coarse graining of polymer dynamics: Simulations guided by statistical beyond-equilibrium thermodynamics. In P. D. Gujrati and A. L. Leonov, editors, Modeling and Simulations in Polymers. Wiley-Interscience, 2010.

[9] I. E. Dzyaloshinskii and G. E. Volovick. Poisson brackets in condensed matter physics. Ann. Phys. (N.Y.), 125(1):6797, 1980 .

[10] H. Stark and T. C. Lubensky. Poisson bracket approach to the dynamics of nematic liquid crystals: The role of spin angular momentum. Phys. Rev. E, 72:051714, 2005.

[11] W. Kung and M. C. Marchetti. Poisson-bracket approach to the dynamics of bent-core molecules. Phys. Rev. E, 76:011710, 2007.

[12] H. Grabert. Projection operator techniques in nonequilibrium statistical mechanics, volume 95 of Springer tracts in modern physics. Springer, Berlin, 1982.

[13] M. Kröger and H. C. Öttinger. Beyond-equilibrium molecular dynamics of a rarefied gas subjected to shear flow. J. Non-Newtonian Fluid Mech., 120:175-187, 2004.

[14] P. Ilg, H. C. Öttinger, and M. Kröger. Systematic time-scale-bridging molecular dynamics applied to flowing polymer melts. Phys. Rev. E, 79:011802, 2009.

[15] P. Ilg and M. Kröger. Molecularly derived constitutive equation for low-molecular polymer melts from thermodynamically guided simulation. J. Rheol., 55:69-93, 2011.

[16] A.M. Luo, L.M.C. Sagis, and P. Ilg. The Landau free energy of hard ellipses obtained from microscopic simulations. J. Chem. Phys., 140:124901, 2014.

[17] A.M. Luo, L.M.C. Sagis, H.C. Öttinger, C. De Michele, and P. Ilg. Modelling the rheology of anisotropic particles adsorbed on a two-dimensional fluid interface. Soft Matter, 11:4383-4395, 2015.

[18] R. F. Snider and K. S. Lewchuk. Irreversible thermodynamics of a fluid system with spin. J. Chem. Phys., 46(8):3163-3172, 1967.

[19] M. Liu and K. Stierstadt. Thermodynamics, electrodynamics, and ferrofluid dynamics. In S. Odenbach (Ed.), Colloidal Magnetic Fluids: Basics, Development and Applications of Ferrofluids. Springer, 2008.

[20] B.J. Edwards, A.N. Beris, and M. Grmela. The dynamical behavior of liquid crystals: A continuum description through generalized brackets. Mol. Cryst. Liq. Cryst., 201: 51-86, 1991.

[21] T.C. Lubensky. Phenomenological dynamics: From Navier-Stokes to chiral granular gases. Pramana-Journal Of Physics, 64(5):727-742, 2005.

[22] H. C. Öttinger. Beyond Equilibrium Thermodynamics. Wiley, Hoboken, 2005.

[23] H. C. Öttinger. General projection operator formalism for the dynamics and thermodynamics of complex fluids. Phys. Rev. E, 57:1416, 1998.

[24] C. Hijón, P. Español, E. Vanden-Eijnden, and R. Delgado-Buscalioni. Mori-Zwanzig formalism as a practical computational tool. Faraday Discussions, 144:301-322, 2010.

[25] P. Ilg. Enhanced Landau-de Gennes potential for nematic liquid crystals from a systematic coarse-graining procedure. Phys. Rev. E, 85(6):061709, 2012.

[26] B. Gupta and P. Ilg. Energetic and entropic contributions to the Landau-de Gennes potential for Gay-Berne models of liquid crystals. Polymers, 5:328-343, 2013.

[27] M. Pavelkal, V. Klika, and M. Grmela. Time reversal in nonequilibrium thermodynamics. Phys. Rev. E, 90(6), 2014.

[28] J. Sinova. Spin Seebeck effect thinks globally but acts locally. Nature Mat., 9:880-881, 2010. 